illness passed off rapidly; but after all fever and constitutional gymptoms had subsided, there remained a very small patch of dulness at the lower part of the right chest. The heart was not displaced, but there was the same paroxysmal cough as I have just referred to. Indeed, so paroxysmal was the cough, that it was suggested that the disease was pertussis, commencing with acute inflammatory symptoms. We determined to explore the dull patch at the base of the lung, and a hypodermic syringe revealed the presence of pus; four drachms were removed. The cough ceased from that moment, and the child was discharged well. Though kept for some weeks under observation there was no return of cough or chest mischief.

Large effusions occurring on the right side of the chest, especially when they simulate solid lung, are doubtless much more difficult to diagnose than when the fluid is on the left side. We do not gain the same help from altered positions of the heart. A large effusion may occur on the right side and the heart beat in its normal position. Of this fact we had striking proof some months past in the case of a poor woman who was admitted under my care. She was sent in for supposed enlargement and disease of liver, that organ being felt below the umbilicus. The enlargement, however, was apparent only, the viscus being displaced downwards by an enormous effusion into the right chest. - Although this effusion was sufficient to influence the position of the liver to such an extent, the apex of the heart was found beating in or about its normal position. A free exit was made for the pus which filled the chest, and the liver returned to within two fingers' breadth of the ribs.

An interesting case occurred to me a few months ago in the case of a strong young man admitted under my care into the West London Hospital. The patient stated that three days before admission he had been seized with a severe shivering fit, and since the attack had had pain over the chest, with difficulty in breathing. The constitutional symptoms were of extreme severity; no physical symptoms of importance could be detected on admission. During the first week it was impossible to examine the chest with exactness, as the patient being in a state of delirious mania, it was with the greatest difficulty he could be kept in bed. There was, however, a plentiful crop of herpes on the lip. and an expectoration of rusty sputa, the balance of clinical evidence being largely on the side of pneumonia. The delirious state was followed by a sharp attack of pericarditis, which again prevented a careful examination of the chest; and it was not till after the patient had been in hospital for nearly a fortnight that we were able to estimate the amount of chest mischief.

Briefly, the physical symptoms were as follows: The right chest was found to be dull, and over this region tubular breathing and bronchophony were well marked. On February 15th, being twenty-eight days after admission, the following note was made: "The general strength of the patient has improved, but the lung has not cleared up. The right chest is sibill dull. Vocal fremitus can be felt on this side, the breathing being tubular. The heart beats in normal position, and the liver is not depressed. The clinical evidence is against the presence of fluid."

$\Lambda$ week later the physical symptoms were much the same. The general condition of the patient was good, his temperature gradually falling. The cough was noted as being peculiar, coming on in whooping-cough-like paroxysms, and unattended with any expectoration. $\AA$ hypodermic syringe detected pus. Subsequently three pints were withdrawn, a free opening being made into the chest, the patient making a complete and rapid recovery. The cough ceased immediately after the pus was removed.

Perhaps nothing has contributed more to the study of medicine as a science than the knowledge gained by an exact investigation into the natural history of individual diseases; in other wordsthe physiology of pathological processes. We should be in a position to state with confidence the time necessary for the lung solidified by pneumonia, to become normal tissue. We should know the relation between such a period and the amount of tissue implicated in the attack. Some such exact knowledge would doubtless help us in forming an opinion as to the presence of fluid in the chest, more especially in those cases where the ordinary symptoms are modified or absent.

Dr. Addison, in his 27th aphorism, writes: "When serous effusion is very considerable, giving rise to unequivocal bronchophony, tubular breathing, want of resonance and rocal vibration, physical examination has repeatedly led to a mistaken belief that these signs resulted from a pneumonia or other consolidation of lung." That similar mistakes may occur, even if the fluid be pus, must be admitted.
In the case of the two patients whose attacks I have briefl brought before you, there was nothing in the course of the illness per se to indicate, even granting the existence of fluid, that that fluid was pus. They had gone through no process sapping the vital forces. They were strong, in vigorous health, and wellnourished. The attacks were marked by extreme severity at the initial outbreak. There was nothing to indicate a septic influence. There was no hectic, no heary sweats, no fluctuating temperature. At the time when the first exploratory puncture was made, in the one case it was normal, in the other $99^{\circ}$; in both cases it had been gradually falling. There was no bulging of ribs, no cedema of parietes, no extreme'stitch-like pain-nothing. I say, to warrant us in thinking the fluid was purulent. Baccelli's test failed in both instances, for in each whispering pectoriloquy xas to be heard with striking intensity. From my own observations I look upon this test as useless, and I have reason for thinking that the vibrations giving rise to the conduction of whispers depend more upon the tension of the fluid than its character. As a general rule tension (that is, distension of the chest) is greater in serous than in purulent effusions: thus we are more likely to hear whispering pectoriloquy in cases of serous effusion. But if, on the other hand, there be a large purulent effusion with consequent great tension, the whispers are as readily conducted as if the fluid were serous.

A paroxysmal cough, more or less of the nature of whooping cough, has been a symptom to which, personally, I owe much help in those cases where the signs of fluid within the chest have been equivocal. My experience is not sufficient to enable me to say with confidence that such a cough, remaining after an acute chest attack has subsided, is invariably indicative or pathognomonic of the presence of fluid. But certainly in nine consecutive cases occurring directly under my own notice such a cough was present, and immediately ceased on withdrawing the fluid from the chest. In all cases the fluid was purulent, and, with one exception, each patient made a complete and speedy recovery, the one exception being a young woman whose attack was evidently septic, and followed a miscarriage.

The case of the little child to which I have very briefly referred was of special interest, the amount of pus removed being but four drachms, this small quantity evidently giving rise to the cough which so closely simulated pertussis.

\section{NOTES ON ANTIPYRIN.}

BY W. TYRRELL BROOKS, M.B.LOND., B.A.OxON., Lichfield Lecturer in Clinical Medicine in the University of Oxford.

Ir is not my intention to make any remarks on the uses of antipyrin as a febrifuge. Antipyrin has been long used for this purpose, long enough indeed for a host of rivals to have arisen, one of which-I mean antifebrin (acetanilide)-bids fair to displace it. I wish rather to bring before the meeting some account of the various diseases for which antipyrin has been used in which it has a more or less specific action, apart from its property of lowering temperature. Antipyrin has been so largely used, during the last year, more especially upon the Continent, that it runs the danger of degenerating into a universal panacea for all ills. So great in fact has been the demand for the drug, that it is believed that the supply has with difficulty kept pace with it, and complaints are now made that the drug is suffering from overpopularity, and that its purity is being sacrificed by the makers to ensure a sufficient quantity in the market.

Antipyrin has been very largely used as an anodyne, and a claim has been made for it by Professors Germain Sée and Lépine that it is a reliable substitute for morphine, whilst in cases where morphine is contra-indicated, such as advanced kidney-disease, acute gout, or certain forms of cerebral irritation, antipyrin may be given freely to allay pain. It has the great advantage over morphine that it does not cause cerebral symptoms ; thus there is not any vertigo nor vomiting, and according to Professor See the use of the drug is not followed by sleep or nervous stimulation. Professor Lepine, however, considers that antipyrin acts both an anodyne and a nerve-stimulant, so that though it relieves pain, it at the same time quickens the intellectual faculties of the patient and renders him disinclined for sleep.

Taking his view of the action of antipyrin as an anodyne, we may say that it is diametrically opposed to morphine in that it

1 Read before the Oxford Branch of the Britinh Medical Aesocintion. 
acts as an anodyne without depressing the higher brain-centres. In only two cases in which I have given antipyrin has it caused sleep, and in these instances I believe the sleep was rather the result of relief from pain than of any somnolent action of the drug. The fact that antipyrin acts as a nerve-stimulant as well as an anodyne is a decided objection to its employment when we wish to relieve pain and at the same time ensure sleep. The best method in such cases is to follow the antipyrin by a hypnotic, such as chloral.

For the immediate relief of pain the drug should be used hypodermically, and as it is very soluble in water, a fresh solution may be made by dissolving one of the tablets prepared by Burroughs and Wellcome in an equal weight of water.

The dose for an adult of antipyrin used hypodermically to relieve pain is fire grains. This has been calculated by Dr. Fränkel of Berlin to be equivalent to one-thirtieth of a grain of morphine. The dose may be repeated if the pain he not relieved. Beyond the pain caused by the injection, and a certain feeling of tension which lasts a fow seconds, no bad effects have been noticed. The drug usually gives relief in from fifteen seconds to half a minute, and the effect lasts for some hours (six to eight hours-Frïnkel).

As an anodyne antipyrin has been used chiefly in herpes zoster. lumbago, ataxia, hepatic and nephritic colic, acute asthma, acute rheumatism, and acute gout.

If given in sufficiently large doses it appears to give relief in the majority of cases. Dr. Fränkel gave it in all cases in which morphine appeared to be indicated, and did not meet with a single failure. Dr. Jennings, of Paris, however, side by side with many cases successfully treated by antipyrin, mentions a case of acute gout which was uninfluenced by the drug.

If given by the mouth as an anodyne antipyrin must be used in large doses: thus Professor Sée recommends a drachm to a drachm and a half in the twenty-four hours, and Professor Lépine 150 grains divided into two doses.

In rheumatism and gout the drug appears to be both sedative and curative in its action; it not only allays the pain, hut in many cases shortens the attack. Professor Sée gave it in fifteen cases of hydrarthrosis which had resisted treatment with the salicylates and also counter-irritation by the actual cautery. In all these cases he found that swelling and pain disappeared in a few days Dr. Fränkel gave it in thirty-four cases, with the result that in all but two there was amelioration of the symptoms and shortening of the attack. In fifteen cases, however, a relapse occurred. He found that the average duration of acute rheumatism with antipyrin was 25 days, whilst with the salicylate treatment it was 35.2 days. Mr. Raymond Johnson tried antipyrin in four cases of acute rheumatism, with the result that it lowered the temperature in all, but in only one out of the four did it relieve the symptoms. The three cases which were unrelieved by antipyrin yielded to treatment with the salicylates, whilst in the fourth case, where salicylate of soda had failed to relieve the patient, antipyrin readily did so.

To give relief in acute rheumatism or acute gout, large doses of antipyrin must be given, 1 to 2 drachms during the twenty-four hours being a usual dose. As a rule the drug produces free sweating and rapid deferrescence. In chronic rheumatism it acts in allaying the pain and shortening the course of the disease. I have given it in a large number of cases of rheumatism, and in the majority I have found it successful. It appears to me to be a remedy which at least should be tried when the salicylates fail or produce disagreeable after-effects, as they occasionally do. Most of the cases recorded in which antipyrin and the salicylate treatment have been used side by side, for the purpose of comparison, yield either to the one or the other, the refractory cases in either section usually yielding to the administration of the other drug. I have not any statistics to prove whether antipyrin is of use in preventing the secondary troubles in acute rheumatism, such as endocarditis.

Antipyrin has been used with great success in nerrous disorders, and I believe it supplies us with a specific for many neuralgic and other allied complaints. Its success in the treatment of migraine and cephalalgia is now assured, and one rarely takes up a medical periodical without finding in it the description of various cases which, after being more or less intractable to remedies for years, have yielded to antipyrin.

In Germany and France especially has this drug been used in the treatment of migraine, and to a less extent in England. During the last few months $I$ have used it in the out-patient department and in private practice in such cases with very good results. As a rule patients return after having taken the remedy, and Task pointedly for some more of the same medicine that they had last time, a fact which stamps its value at once on one's mind.

In treating migraine with this drug, I believe the best plan is to use the remedy as a specific against the attacks, and not to administer it continuously. If the migraine be periodic, or if there be a preliminary aura, the drug should be exhibited as soon as possible before the threatened attack. Thus, if an attack bo feared for the morning, antipyrin should be given at night, and if the attack still threatens in the morning, a further dose should be administered. In this way the attack generally is aborted. Even if preliminary warning be absent, the medicine taken as soon as the attack comes on either aborts it, or renders its symptoms lew intense. In my experience it is very rare for antipyrin to fail to influence farourably an attack of migraine, and in this I am supported by almost all of those who have noted on this drug.

It is rarely necessary to give large doses to produce the specific effect. I generally give 5 to 7 grains combined with alkalies and a bitter infusion, to be taken when an attack threatens, and to be repeated, if necessary in an hour. I find that somewhat larger doses are recommended ( 15 to 20 grains), but patients rarely complain that the smaller dose fails.

I have found the drug useful also in those cases of bilious headache, which often occur in patients of full habit, who are addicted to the too frequent use of alcohol. These cases, which generally occur amongst women in a comfortable position in life, yield to the administration of antipyrin; I had the satisfaction of hearing a patient, who has suffered in this way for more than ten years, state that at last a remedy had been found which relieved her. Of course the remedy does not touch the root of the evil.

In some cases of cephalalgia, antipyrin relieves for a time, but at length the patient becomes habituated to the drug, and the relief is less marked. In such cases, either the drug may be increased, or antifebrin or some other of the substitutes for antipyrin may be used.

As antipyrin has so marked an influence orer these nervons complaints, it seems natural to suppose that it may be useful in epilepsy.

Fraty concludes that it has a distinct influence over epilepery akin to that manifested by the alkaline bromides, but he confesees that large doses must be given ( 1 to 2 drachms daily), and that in a considerable number of cases it has to be given up, owing to the malaise it produces.

I have not tried the drug in many cases of epilepsy, but I was not favourably impressed with the result when I did try it. As sedative antipyrin has been tried in cases of nocturnal emissions, and it has been found that 7 to 15 grains administered on going to bed prerents the emission in many cases. It also acts in diminishing the excessive flow of urine which not infrequently accompanies spermatorrhœa, and which arises from the hyperæsthesia of the nerrous system. I would venture to think that this druo may be well worth a trial in those cases which so often are found to exist in young men who have fallen into the habit of masturber tion at school, and who, on coming into the world, learn the evile of it, and relinquish the habit, but in whom spermatorrhoes froquently supervenes to a serious extent. I have given it in similar cases with good results, the best plan being to give 10 grains of antipyrin in combination with 10 grains of chloral hydrate at bed time, the patient usually falling asleep shortly after getting into bed, and remaining asleep without disturbance till the morning

Antipyrin was given by $\mathbf{M}$. Bloch to a neurotic man with a tender spine, who was periodically orercome by attacks of drowsiness, which came on after each meal ; these were accompanied by pains in the head and debility. His condition had been improved by the use of nux vomica to some extent; but, on the exhibition of antipyrin in 15-grain doses given on waking and at 11 A.M., the drowsiness after a few days disappeared, and the remaining nerrous symptoms abated. In this case it acted as a decided nerve stimulant.

The drug has been strongly recommended in cases of chorea by Legroux, who considers it a most rapid, certain, and inoffensive remedy. He administered it in six cases, giving 40 to 50 grains daily. All his cases recovered rapidly in from 6 to 27 days. I have not had the opportunity to use it frequently in chorea, but in such cases as I have used it the movements diminished rapidly. In one child to whom I gave the drug it had to be discontinued, owing to the cardiac depression which accompanied its use.

Antipyrin has been used with success in spasmodic nervous disorders such as hay ferer and whooping-cough. Dr. Bloch tried it 
in a case of hay fever in which cocaine and the bromides had been given without result. He gave it in 30 -grain doses at the hours when the attacks usually came on, and found that the drug abiorted the attacks. After taking antipyrin for some weeks the disease disappeared in this case.

Sonnenberger, from an experience of 70 cases in which he used the drug in whooping-cough, concludes that it is a very useful remedy in such cases. He gave it to infants in doses of $\frac{1}{2}$ to $1 \frac{1}{2}$ grain three times a day in syrup of tolu or raspberry, increasing the dase to 10 or 15 grains for older children. The remedy must be used systematically to produce a good result in whooping-cough.

In nervous vomiting, especially in the vomiting of pregnancy, antipyrin is useful. If the vomiting be periodic, the drug should be given a few hours before the usual appearance of the attack. In sea-sickness the drug has been lauded as a specific, perhaps only to have its day as most other specifics for this disorder have had. More than one medical man has, however, recorded the debt of gratitude he owes to this remedy in crossing the Atlantic, so that it may be tried in the hope that it may be of use.

Antipyrin has been used as a hæmostatic in cases of pulmonary hemorrhage by Dr. Olikoff. He made a solution of 15 grains to the ounce in water, and made his patients breathe through this for four or five respirations, repeating the use of it every half hour. In all the six cases tried the hæmorrhage was diminished. As a hæmostatic for general purposes, antipyrin is too costly a remedy to be employed lavishly, though it has been recommended for epistaxis and other forms of hæmorrhage. Herpes zoster and locomotor ataxy have both been successfully treated with antipyrin. In locomotor ataxy it appears to act in alleviating the lightning pains and in giving ease to the patient rather than by altering the counse of the malady.

Since antipyrin became a popular remedy, many cases in which the drug has produced disagreeable effects have been recorded, though, as far as I am aware, none of these cases have ended fatally, nor have there been any symptoms which have lasted more than a few hours. The cases which I have collected (more than twelve in number) appear to me to be pure examples of idiosyncrasy. They are usually isolated cases occurring amidst many others in which the same quantity of the drug was administered. They do not appear to depend on the quantity of the :drug given, for in one case 4 grains, in another 8 grains, and in a third 15 grains of antipyrin produced symptoms of poisoning, though more than double the dose has been given in many hundreds of cases without bad effects. There is, as far as I can find, no special class of cases in which the administration of antipyrin is likely to bring on symptoms of poisoning; but, as it appears in certain individuals to cause disagreeable symptoms, regardless of dose, we are likely to hear further of this property it possesses from some of the large number of people who are now taking the drug as a preventive against sea-sickness.

The chief symptoms which manifest themselves in cases of poisoning by antipyrin are certain nervous sensations, such as restlessness, loss of memory, a feeling of general expansion of the body, and a sensation of great coldness. These are followed by swelling of the face and the appearance of an erythematous eruption resembling measles-so much like it, in fact, that those who have seen cases of antipyrin rash are careful to warn us to avoid the diagnosis of measles in patients taking antipyrin.

The chief points of difference between this rash and measles are that it appears but slightly on the face, that its chief distribution is on the extremities, that it is non-crescentic in distribution. In many cases it is not accompanied by catarrh of the eyes and nose, but in a few cases catarrh does occur, and when present it must make the differential diagnosis very difficult. Besides these symptoms, antipyrin may cause diaphoresis, feebleness of the pulse; and general collapse. Gastro-enteritis occurs rarely.

The antidote which removes these disagreeable effects most rodily is belladonna, given either as the tincture or in the form of atropine used hypodermically (one-seventieth of a grain).

Conclusions.-I would venture to think that in antipyrin we have a drug which, though suffering from a temporary overpopularity, is likely to be of use in practice. Its power of relieving migraine and other forms of cephalalgia is, in many cases, magical. As an anodyne it is particularly useful in those cases wheremorphin is contra-indicated, especially in advanced kidrey disease, acute gic.t, or in the bronchitis of old people. I do not think that antipyrin is at all likely to displace morphine, as this "drug possesses the advantage of being much more powerful bulk for bulk, and hence is more convenient for hypodermic medica- tion. But a trial of it should be made where morphine cannot be given, or where morphine must be withheld for fear of establishing the morphine habit. In cases of long continued pain in which some anodyne must be given for a period often stretching over years, antipyrin may be found useful as an occasional substitute for morphine when the patient has become habituated to the morphine, and when it otherwise would be necessary to increase the dose of this drug. I regret I have not met with a case in which I could try this, but such cases as locomotor ataxy, or cases of slow paralysis accompanied by spasm of the muscles, would be suitable ones in which to make a trial.

I do not think antipyrin will displace the alkaline salicylates in the treatment of acute rheumatism, but it is undoubtedly useful where the salicylates have failed, or where they are contraindicated by the disagreeable effects they occasionally produce.

With regard to the objection raised against antipyrin that it not infrequently gives rise to symptoms of poisoning, I believe that such is of little value. I have given the drug in a large number of cases without meeting with any bad effects from it, and few of those who have used this drug most largely lay any stress upon this difficulty. One must be prepared to meet with cases of idiosyncrasy in the administration of this drug as one has to be with cocaine, morphine, quinine, and other drugs.

By far the most sericus objection to its extended use, particularly in hospital practice, is expense. At present, its manufacture is in the hands of monopolists, and though the French chemists say they have ascertained its composition and method of preparation, no one at present has sent on the market any of the drug under its proper chemical name, which is dimethyl-oxiquinisin, a name which requires some reflection before being added to a prescription

I have tried antifebrin as a substitute for this drug in several cases of migraine, and though the effect does not seem so certain as when antipyrin is used, yet in many cases it has acted well. The relative expense of antifebrin is much less than that of antipyrin.

\section{THE USE OF "SALUFER" (SILICO-FLUORIDE OF SODA) AS AN ANTISEPTIC.}

BY A. W. MAYO ROBSON, F.R.C.S.,

Honorary Surgeon, Leeds General Infirmary ; and Lecturer on Practical Surgery at the Yorkshire College.

A PERFECT antiseptic for surgical purposes has yet to be found. It must be at the same time a strong germicide, non-poisonous, unirritating to the skin or to the tissues, inodorous, non-volatile, not destroyed by oxidation, non-corrosive to steel instruments, non-injurious to sponges, and inexpensive. In "salufer" (silicofluoride of sodium) we have a substance which would seem to fulfil most of these conditions. Mr. W. Thomson, F.R.S.Edin., read a paper before the British Association at Manchester last year in which he explained the results of certain experiments he had made with respect to the antiseptic properties of some of the fluorine compounds. He had tried the effects, on flour paste and on meat chopped into small pieces and mixed with water, of a very large number of chemical compounds, and had found that those which had the most remarkable antiseptic properties were the compounds of fluorine, the neutral fluorides of sodium, potassium, and ammonium, and their fluosilicates. Fluosilicate of soda he found to be the best adapted for use as an antiseptic, it being non-poisonous, inodorous, and sparingly soluble in water. As it had only a very slightly saline taste, he said it might be employed in preserving food without communicating any taste to it. Mr. Thomson stated that a saturated solution which contained only 0.61 per cent. possessed greater antiseptic power for animal tissue than 1 in 500 perchloride of mercury solution.

For general surgical purposes I have been very well satisfied with perchloride of mercury, which is a most efficient antiseptic, and which is so very conveniently carried in the form of powders, each containing five grains of perchloride and five grains of salammoniac, which quantity, dissolved in a pint of water, makes a 1 in 2,000 solution : but it has the great disadvantages of roughening and cracking the hands, of corroding steel instruments, and of being poisonous. Hence it is dangerous for syringing out large cavities-for example, psoas abscess; for washing out serous sacs -for example, peritoneum and pleura; or for irrigating the interior of the uterus after gynæcological operations or in obstetric practice. 